5. Public Law: Prenatally and Postnatally Diagnosed Conditions Awareness Act of 2008, Pub. L. No. 110-374 (8 October 2008).

6. Skotko BG, Levine SP, Goldstein R. Having a son or daughter with Down syndrome: perspectives from mothers and fathers. Am J Med Genet Part A 2011;155:23335-23347.

7. Skotko BG, Levine SP, Goldstein R. Having a brother or sister with Down syndrome: perspectives from siblings. Am J Med Genet A 2011; 155A:2348-2359.

8. Skotko BG, Levine SP, Goldstein R. Self-perceptions from people with Down syndrome. Am J Med Genet A 2011; 155A:2360-2369.

doi:10.1038/gim.2011.58

\section{Medical genetics and genomics curricula focused on the laboratory specialties}

To the Editor: Medical genetics is a rapidly growing medical specialty that offers unique opportunities for $\mathrm{PhD}$-degreed scientists to become board certified and participate in clinical activities, most notably by directing diagnostic laboratories. However, in our experience, few $\mathrm{PhD}$ students are aware of medical genetics as a career path and most lack an understanding of the environments in which medical geneticists work and the training requirements that lead to eligibility for certification by the American Board of Medical Genetics in a laboratory specialty. To raise awareness about medical genetics as a career path among graduate students and postdoctoral fellows at Baylor College of Medicine, two curricula focused on the laboratory specialties of medical genetics have been developed for the Graduate School of Biomedical Sciences. These curricula complement the Genetics Track Curriculum for medical students that was described by Dhar et al. in their article "Enhancing Exposure to Genetics and Genomics Through an Innovative Medical School Curriculum."

A medical genetics internship was developed to introduce medical genetics as a career path and define the work environments and training requirements associated with medical genetics. The internship consists of some components of the Genetics Track Curriculum, including an online human subjects research training course, case-based genetics conferences, journal clubs, and mentorship meetings. Internship participants also attend a "Careers in Medical Genetics" informational seminar and participate in laboratory rotations that include 1 week each in the Biochemical Genetics, Cytogenetics, and Molecular Genetics divisions of the Baylor College of Medicine Medical Genetics Laboratories. The internship requirements must be completed in 12 months. Assessment is attendance based and those completing the program receive a certificate of completion.
The internship debuted in September 2011. Interest far exceeded any capacity of the Baylor laboratories to accommodate students on rotations. As a result, a course titled "Introduction to Medical Genetics" was developed to replace the internship after the 2011-2012 academic year. The course includes 11 lectures that will define the specialty of medical genetics and its place in the practice of medicine in the United States; outline educational and training requirements for eligibility for certification by the American Board of Medical Genetics in a laboratory specialty; introduce technologies used in medical genetics laboratories; explore topics such as pharmacogenetics and cancer diagnostics; describe the regulatory environments in which clinical laboratories operate; discuss billing and reimbursement, quality control/assurance, and other operational considerations unique to clinical laboratories; delineate how clinical and analytic sensitivity/specificity and clinical utility of genetic tests are assessed; consider how copy-number and sequence variations are evaluated for clinical significance; and introduce ethical and legal issues relevant to medical genetics. Students will also complete an online human subjects training course and participate in two small group discussions focused on cases from biochemical genetics, clinical genetics/genetic counseling, cytogenetics, and molecular genetics.

In our experience, medical geneticists have only to reach out to students and postdoctoral fellows to identify individuals interested in medical genetics as a career path. It is our hope that the Genetics Track Curriculum, medical genetics internship, and/or "Introduction to Medical Genetics" course may be replicated at other institutions. Currently, we are considering how to disseminate the course materials for use by others.

\section{ACKNOWLEDGMENTS}

The authors are grateful to Shweta U. Dhar, Christine Eng, Nancy Moreno, Lorraine Potocki, and Frank Probst for their contributions to the internship and/or course.

\section{DISCLOSURE}

The authors declare no conflict of interest.

\section{Ping Fang, $P h D^{1}$ and Raye L. Alford, $P h D^{2}$}

${ }^{1}$ Department of Molecular and Human Genetics, Baylor College of Medicine, Houston, Texas, USA; ${ }^{2}$ Bobby R. Alford Department of Otolaryngology-Head and Neck Surgery, Baylor College of Medicine, Houston, Texas, USA. E-mail: ralford@bcm.edu

\section{REFERENCE}

1. Dhar SU, Alford RL, Nelson EA, Potocki L. Enhancing exposure to genetics and genomics through an innovative medical school curriculum. Genet Med 2012;14:163-167. 